\title{
Endoscopic ultrasound-guided tissue acquisition of pancreatic masses
}

\author{
Ihab I. El Hajj, MD, MPH, Mohammad Al-Haddad, MBBS, MSc* \\ Division of Gastroenterology and Hepatology, Indiana University School of Medicine, 550N. University Blvd, Suite 4100, Indianapolis, Indiana
}

\section{A R T I C L E I N F O}

\section{Article history:}

Received 9 August 2017

Accepted 7 January 2018

\begin{abstract}
A B S T R A C T
Endoscopic ultrasound (EUS) has assumed an increasing role in the management of pancreaticobiliary disease over the past 2 decades but its impact is particularly evident in the management of pancreatic masses. EUS helps improve patients' outcomes by enhancing tumor detection and staging while providing safe and reliable tissue diagnosis. This review provides an evidence-based approach to the use of EUS for the diagnosis of pancreatic cancer, its staging, and for the determination of resectability compared to other imaging modalities. We will focus on techniques specific to obtaining tissue from solid pancreatic masses and will review best practices in EUS-guided tissue acquisition.
\end{abstract}

(c) 2018 Elsevier Inc. All rights reserved.

\section{Introduction}

Advancements in radiologic and endoscopic ultrasound (EUS) imaging have improved our ability to detect and stage pancreatic masses allowing for more selective surgical intervention for patients with "resectable disease." Owing to the low sensitivity of cross-sectional imaging to detect small tumors in the pancreas, endoscopic diagnosis by using EUS has become a mainstay for the assessment of pancreatic masses. EUS also provides a reliable method for tissue sampling hence securing a histopathologic diagnosis [1-3]. This review will focus on the role of EUS in the evaluation of pancreatic masses compared to other imaging modalities, and highlights the best practices to improve tissue yield from EUS-guided tissue acquisition (EUS-TA).

\section{Pancreatic cancer}

\subsection{Background and epidemiology}

Pancreatic cancer is the fourth leading cause of cancer-related mortality in the United States. Over 45,000 patients are diagnosed each year in the United States, and the majority of these patients succumb to their disease [4]. Eighty percentage of patients are diagnosed with advanced, unresectable disease. According to the latest statistics, only $7 \%$ of patients survive 5 years after diagnosis [4]. While the 5-year survival rate improves to $25 \%$ in patients presenting with stage 1 or localized disease, only $9 \%$ of patients are

\footnotetext{
Research support for Mohammad Al-Haddad from Boston Scientific.

* Corresponding author.

E-mail address: moalhadd@iu.edu (M. Al-Haddad).
}

identified at this early stage. The majority of patients (53\%) presents with distant, metastatic disease, and have a 5-year survival of $2 \%$. Identification of risk factors and establishing earlier detection methods are therefore of paramount importance [5].

\subsection{Cross-sectional imaging}

\subsubsection{Computed tomography}

Computed tomography (CT) is the most widely used imaging modality for the assessment of suspected pancreatic ductal adenocarcinoma (PDAC). CT imaging has significantly improved with the introduction of multiple-detector CT (MDCT), which allows high-resolution and multiplanar image reconstruction. CT is reported to have a sensitivity of $89 \%-97 \%$ for PDAC, though it is less effective in diagnosing small $(<2 \mathrm{~cm}$ ) lesions with a sensitivity of $65 \%-75 \%$ [6]. In this respect, EUS is superior in tumor detection. Comparative studies between EUS and MDCT for pancreatic tumors have demonstrated the superiority of EUS for tumor detection compared to multirow CT. Agarwal et al [7] reported an EUS sensitivity of $100 \%$ for the diagnosis of cancer compared to $86 \%$ for MDCT. Similarly, DeWitt et al [8] reported that the sensitivity of EUS (98\%) was statistically superior to MDCT (86\%) in a cohort of 80 patients with pancreatic cancer.

\subsubsection{Magnetic resonance imaging}

Contrast-enhanced magnetic resonance imaging (MRI) has a sensitivity and accuracy at least similar to that of MDCT for diagnosis and staging of pancreatic cancer, but it is costlier and less readily available than MDCT. MRI, however, may more reliably detect smaller, non-contour-deforming tumors compared with CT [9]. MRI also more accurately detects and characterizes smaller hepatic metastases [10]. A recent study concluded that MRI was 
superior to CT for tumor detection but performed similarly for the evaluation of resectability [11]. In a study that compared the diagnostic performance (detection, local staging) of multiphasic 64-detector CT with gadobenate dimeglumine-enhanced 3.0-T MRI in patients suspected of having pancreatic cancer, both CT and MRI were found to be equally suited for detecting and staging pancreatic cancer [12]. Therefore, the choice of imaging modality for detection and staging of pancreatic cancer depends on test availability and local expertise.

\subsubsection{Positron emission tomography and integrated PET/CT}

The role of functional imaging especially positron emission tomography with 2-deoxy-2-[fluorine-18]fluoro-D-glucose integrated with CT (FDG-PET/CT) is still uncertain in the staging of pancreas cancer. The NCCN guidelines list the possible performance of PET/CT for the detection of regional lymph nodes and extrapancreatic metastases, although it has not been incorporated in routine practice [13]. The sensitivity and specificity of FDG-PET/ $\mathrm{CT}$ in the diagnosis and evaluation of pancreas cancer ranges from $71 \%-100 \%$ and 64\%-95\%, respectively, significantly higher than those of CT alone $[14,15]$. The sensitivity of PET/contrast-enhanced CT in detecting local recurrence, abdominal lymph node metastasis, and peritoneal dissemination are 83\%, 88\%, and $83 \%$, respectively [16]. A meta-analysis of 51 studies involving 3857 patients compared the diagnostic performance of ${ }^{18}$ FDG PET alone, ${ }^{18}$ FDG PET/CT, and EUS for diagnosing pancreatic cancer [17]. The study concluded that the pooled sensitivity for combined PET/CT (90.1\%) was significantly higher than PET (88\%) and EUS (81\%). However, the pooled specificity estimate for EUS (93.2\%) was significantly higher than PET (83\%) and PET/CT (80\%).

\subsection{Staging of pancreatic adenocarcinoma}

Staging of pancreatic cancer is performed according to the American Joint Committee for Cancer (AJCC) Staging TNM classification, which describes the tumor extension $(\mathrm{T})$, lymph node $(\mathrm{N})$, and distant metastases (M) of tumors, respectively [18]. The accuracy of EUS for T staging of pancreatic tumors ranges from 62\%-94\% [19-21]; while its accuracy for $\mathrm{N}$ staging ranges from $41 \%-86 \%$ [5].

Para-aortic lymph nodes (PALNs) are considered nonregional lymph nodes for both pancreatic head and body or tail cancers, thus meticulous survey of this region is critical during staging of all pancreatic tumors [22]. Kurita et al [23] conducted a prospective, nonrandomized single-center trial, of 208 patients with pancreatobiliary cancers without apparent distant metastases except for PALNs. PET/CT and EUS-guided fine-needle aspiration (EUS-FNA) were performed sequentially as a single combined procedure to evaluate PALN metastasis. EUS-FNA had higher sensitivity, specificity, positive predictive value, negative predictive value, and accuracy for the diagnosis of PALNs metastasis than PET/CT. The differences for the sensitivity and accuracy were significant $(P<$ 0.001). An EUS survey of mediastinal stations for metastatic adenopathy is also warranted since these are also considered nonregional lymph nodes.

For detection of nonnodal metastatic cancer, CT and MRI are superior to EUS due to both anatomical considerations of the upper gastrointestinal tract and the limited range of EUS imaging. However, EUS still has an important role in the evaluation of 1 hepatic metastasis in the left or caudate lobe (Figure 1) and malignant ascites, some of which can be missed on cross-sectional imaging and both of which can be accessible by EUS-FNA. Identification of liver metastases or malignant ascites by EUSFNA may preclude surgical resection and is associated with poor survival following diagnosis [24].

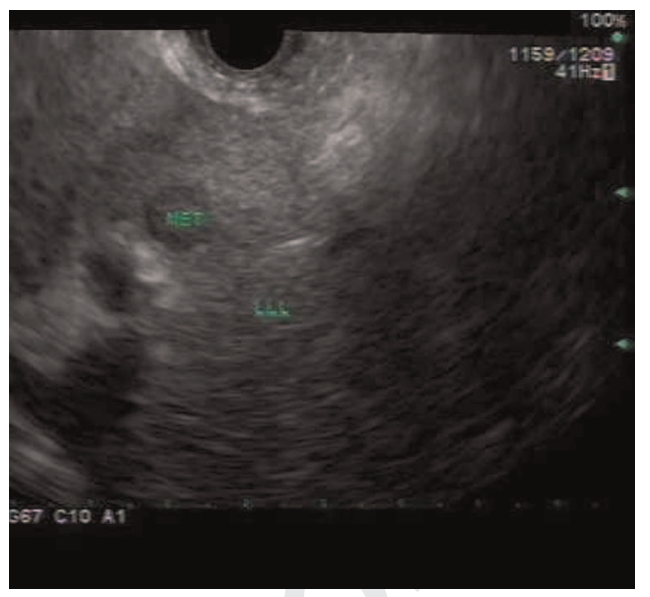

Fig. 1. A linear EUS image of a small liver lesion not visualized on CT scan in a patient undergoing staging and FNA of a pancreatic body mass. Cytology from the lesion confirmed metastatic pancreatic adenocarcinoma. (Color version of figure is available online.)

\subsection{Assessment of vascular invasion}

The overall accuracy of EUS for vascular invasion ranges from $68 \%-93 \%$ [19,25-27]. The overall accuracy of CT is reportedly equivalent $[19,26]$ or inferior [25] to EUS. The overall accuracy of MRI is reportedly equivalent [19] or superior [26] to EUS.

The overall sensitivity and specificity of EUS for malignant vascular invasion range from $42 \%-91 \%$ and $89 \%-100 \%$, respectively [19,25-27]. The sensitivity of EUS for tumor invasion of the PV or porto-splenic confluence is $60 \%-100 \%[28,29]$ with most studies demonstrating sensitivities over $80 \%$. The sensitivity of EUS for PV invasion (Figure 2) is consistently superior to that of CT $[28,30,31]$. For the superior mesenteric vein, superior mesenteric artery (Video 1), and celiac artery, the sensitivity of EUS is $17 \%-83 \%$ [27], 17\% [32], and about 50\% [28], respectively. The sensitivity of CT for staging of the superior mesenteric artery [31,32] and celiac artery [28] appears to be better than EUS. Until further conclusive data becomes available, assessment of tumor resectability should be done by both EUS and CT (or MRI) rather than by EUS alone.

\subsection{Resectability of pancreatic tumors}

In a pooled analysis of 9 studies involving 377 patients, the sensitivity and specificity of EUS for resectability of pancreatic cancer was $69 \%$ and $82 \%$, respectively $[8,19,25-27,33-36]$. The

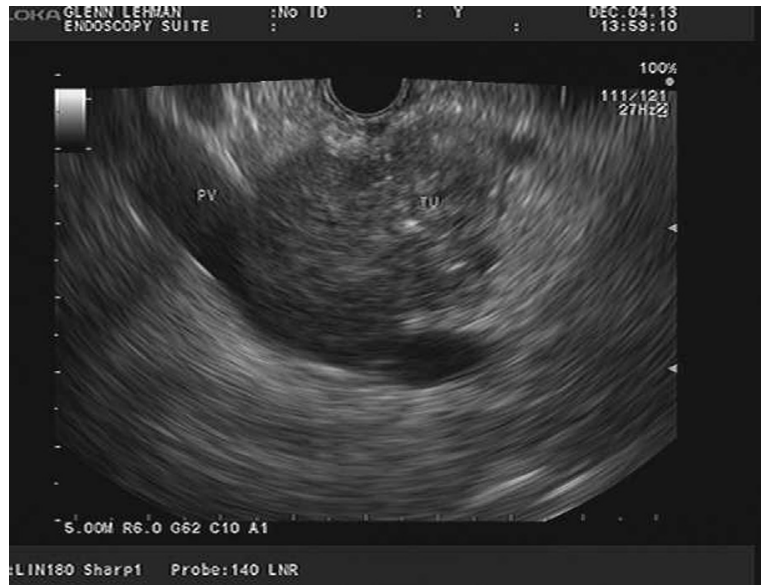

Fig. 2. A linear EUS image of a pancreatic head mass invading the portovenous confluence. This patient underwent neoadjuvant therapy to downstage the tumor followed by pancreaticoduodenectomy with venous reconstruction. 
accuracy of EUS for tumor resectability was 77\%. Using a decision analysis, Soriano et al [19] found that accuracy for tumor resectability was maximized and costs were minimized when CT or EUS was performed initially followed by the other tests in those with potentially resectable neoplasms. Ahmad et al [33] proposed that although EUS and MRI individually are not sensitive for tumor resectability, their combined use may increase positive predictive value of resectability compared to either test alone. When surgery is performed only when MDCT and EUS agree on tumor resectability, DeWitt et al [8] reported a nonsignificant trend toward improved accuracy of resectability compared to either study alone. However, a study by Bao et al [37] found that MDCT was a better predictor of resectability than EUS. In the recent years, higher resolution assessment for vascular invasion and distant metastasis by multiphasic CT has assumed a larger role than EUS in determining surgical candidacy of patients with pancreatic cancer.

\subsection{EUS-guided tissue acquisition of pancreatic cancer}

EUS-FNA remains the first-line modality for tissue sampling in patients with pancreatic masses [38,39]. Based on the results of 2 meta-analyses [40,41], the pooled sensitivity and specificity of EUS-FNA for diagnosis of pancreatic adenocarcinoma ranged between $85 \%-89 \%$ and $96 \%-98 \%$, respectively. The presence of chronic pancreatitis may impair the visualization of tumors endosonographically or hinder the cytologic interpretation of the sampled pancreatic tissue, thus reducing sensitivity. In a series of 207 consecutive patients with focal pancreatic lesions, FritscherRavens et al [42] found that the sensitivity of EUS-FNA for the diagnosis of malignancy in patients with normal parenchyma to be superior (89\%) to those with parenchymal evidence of chronic pancreatitis (54\%).

Today, EUS-TA by FNA (EUS-FNA) and fine-needle biopsy plays a pivotal role in the diagnosis of pancreatic masses. Obtaining an adequate sample and reaching an accurate diagnosis are fundamental endpoints of EUS-TA [39]. This is of particular importance since many patients with malignancy are often subjected to neoadjuvant systemic therapy prior to surgery nowadays, where a tissue diagnosis is essential to move this process forward. Significant efforts have been made in recent years to identify the ideal EUS-TA technique, one that is efficient, effective, and associated with high diagnostic yield, specimen adequacy, accuracy, and low adverse event rate [43]. These efforts have focused on studying several variables associated with EUS-TA outcomes and can be categorized as: (1) those related to sampling methods and techniques (use of suction and stylet, fanning and capillary technique, number of passes, methods of sample expression); (2) availability of rapid on-site evaluation (ROSE), (3) endosonographer and cytopathologist qualifications (experience, training, and competency); and (4) type of specimen and needle used. We will expand on each one of these variables in the subsequent sections of this review.

\subsubsection{Sampling methods and techniques}

2.6.1.1. Use of suction vs capillary suction technique. Use of air vacuum (suction) remains widely practiced during FNA of a variety of solid and cystic lesions. Suction is generally recommended for pancreatic solid lesions, particularly PDACs which can carry a variable degree of stromal fibrosis and desmoplasia. In highly vascular lesions such as lymph nodes and neuroendocrine tumors, a nonsuction technique is recommended allowing for a better quality and less bloody sample. Avoiding suction in vascular lesions can improve the quality of ROSE with less blood that could interfere with tumor visualization; however, in passes dedicated solely for cell block, suction may be reintroduced to improve tissue acquisition.

The wet suction technique (WEST) relies on preflushing the needle with saline to replace the column of air with fluid followed by applying negative pressure on the proximal end of the needle. In a prospective, single-blind, randomized, controlled trial using a 22-gauge needle for EUS-FNA of solid lesions, WEST resulted in significantly better cellularity and specimen adequacy in cell blocks of EUS-guided FNA aspirate of solid lesions than the conventional FNA technique [44]. A new modified WEST (hybrid suction technique) relies on preloading the needle with saline, but having continuous negative pressure with a prevacuum syringe to avoid manual intermittent suction. Data about this technique is limited to a single-center pilot study by Berzosa et al [45]. Another recent randomized controlled trial showed that high negative pressure suction (generated by using a $60-\mathrm{mL}$ syringe) was associated with superior diagnostic yield compared to standard negative pressure using a $10-\mathrm{mL}$ syringe in patients with pancreatic masses undergoing EUS-FNA [46]. In our practice, we continue to use suction during aspiration of solid pancreatic masses when collecting for cell block but would limit its use when the on-site review from the initial pass indicates large amounts of blood and paucity of tumor cells.

Capillary suction technique utilizes capillary aspiration created by slow and staggered withdrawal of the stylet. This has been suggested in limited studies to enhance quality of the specimen obtained for diagnostic purposes $[47,48]$. Based on 1 study, this technique was associated with better cellular quality and diagnostic yield in pancreatic and liver masses [49].

2.6.1.2. Use of stylet. The presence of a stylet should prevent the introduction of gastrointestinal wall tissue to the needle as it traverses this to access the target lesion. However, current data suggest that the use of a stylet does not confer any advantage during EUS-FNA [50]. Furthermore, the use of stylet is considered to be labor intensive and time consuming (particularly with $25 \mathrm{G}$ needles), which could prolong procedure time and theoretically increase the risk of inadvertent needle injuries in the endoscopy suite.

2.6.1.3. Needle size. Current EUS-FNA needles are available in 25 gauge, 22-gauge, and 19-gauge needles. Needle size is probably the most widely studied factor as a predictor of cytologic adequacy and diagnostic yield of malignancy. The 22-gauge needle were considered the default needle for a long time but a recent reduction in its utilization has been described in favor of 25gauge needles, particularly when sampling pancreatic head and uncinate process lesions. To date, 3 meta-analysis compared the diagnostic accuracy of EUS-FNA for pancreatic masses by using 22 and 25-gauge needles demonstrated superior sensitivity of 25gauge needles for diagnosing pancreatic malignancy [51-53]. In addition, randomized controlled trials suggest that there is no incremental diagnostic yield of $19 \mathrm{G}$ vs 22 or $25 \mathrm{G}$ with overall similar safety profile $[54,55]$. Table 1 summarizes the studies comparing the diagnostic yield of malignancy between $22 \mathrm{G}$ and $25 \mathrm{G}$ needles during EUS-FNA of pancreatic masses [56-61].

2.6.1.4. Fanning technique. The fanning technique for EUS-FNA involves sampling multiple areas within a lesion by changing the angle of the tip of the scope or (when smaller gauge needles are used) by using the elevator. Bang et al [62] compared this technique to the standard technique for EUS-FNA of solid pancreatic mass lesions, and found fanning to be superior by establishing a diagnosis in fewer passes, and resulted in higher first pass diagnostic rate ( $86 \%$ vs $58 \%$; $P=0.02$ ). While further data 
Summary of studies comparing diagnostic yield of malignancy between 22-gauge and 25-gauge needles during EUS-FNA of pancreatic masses.

\begin{tabular}{llccr}
\hline Author & Study design & No. of patients 22G/25G & Sensitivity (95\% CI) 22G & Sensitivity (95\% CI) 25G \\
\hline Siddiqui et al [56] & RCT & $64 / 67$ & $0.88(0.77-0.94)$ & $0.96(0.87-0.99)$ \\
Yusuf et al [57] & Retrospective & $540 / 302$ & $0.84(0.80-0.88)$ & $0.92(0.87-0.99)$ \\
Siddiqui et al [58] & Retrospective & $26 / 17$ & $0.85(0.62-0.97)$ & $0.91(0.59-1.00)$ \\
Camellini et al [59] & RCT & $43 / 41$ & $0.86(0.70-0.95)$ & $0.89(0.75-0.97)$ \\
Uehara e al [60] & Retrospective & $54 / 66$ & $0.88(0.74-0.96)$ & $1.00(0.91-1.00)$ \\
Fabbri e al [61] & Prospective & $50 / 50$ & $0.85(0.71-0.94)$ & $0.94(0.82-0.99)$ \\
\hline
\end{tabular}

Abbreviations: G, gauge; RCT, randomized controlled trial.

(Adapted with permission from Wani et al [39]). pancreatic lesions.

\subsubsection{Rapid on-site evaluation}

The availability of a cytopathologist on site (for ROSE) has been shown to improve the diagnostic yield of EUS-FNA for malignancy. Prior studies demonstrated a $10 \%-15 \%$ increase in diagnostic yield in the presence of ROSE and $20 \%$ rate of nondiagnostic aspirates in its absence $[70,71]$. The beneficial role of ROSE for EUS-FNA of solid pancreatic masses was confirmed by a meta-analysis of 34 studies (3644 patients) [70]. However, recent data from 2 multicenter trials showed no significant difference in the diagnostic yield of malignancy, proportion of inadequate specimens, and accuracy in patients with pancreatic mass undergoing EUS-FNA with or without ROSE [71,72]. In these studies, ROSE was associated with fewer number of passes. It has been suggested that ROSE may have a role only during the learning phase of EUS-FNA in recently established EUS services. In our practice, we find ROSE to be of significance in limiting the number of passes and hence improve procedural efficiency and reduce patient risk. It also allows a real-time decision to be made on whether additional tissue assays are needed (such as microbiology studies, flow cytometry, and molecular assays). In the absence of ROSE, it is recommended performing 4-5 passes in solid pancreatic lesions and 2-3 passes in lymph nodes, liver, and adrenal lesions.

The interobserver variability among cytopathologists with regard to EUS-FNA and fine-needle biopsy specimens is an issue that has significant implications for patient management. In a pilot study, Mounzer et al [73] evaluated the interobserver variability among 4 cytopathologists in assessing EUS-FNA cytology specimens of solid pancreatic lesions using a novel standardized scoring 4 system. The study demonstrated that the interobserver agreement improvement when combining suspicious and malignant diagnoses $(\kappa=0.54)$. Similar to recent advances in EUS performance secondary to improved training and better competence and quality metrics assessment [74], cytopathologists need to address these critical issues in cytology performance in future studies. is awaited, we routinely perform fanning during sampling of solid for the final diagnosis was moderate $(\kappa=0.45)$ with minimal

\subsubsection{Type of specimen}

EUS-FNA remains the standard procedure for sampling of pancreatic masses. However, EUS-FNA has certain limitations. First, primary pancreatic lymphomas (PPLs) and well-differentiated ductal adenocarcinomas are often difficult to diagnose by use of cytology alone. Second, chronic pancreatitis, if present, can obscure the detection of pancreatic tumors and hinder a cytological diagnosis of malignancy. Third, the low negative predictive value of EUS-FNA does not permit exclusion of malignancy in negative specimens. To address these limitations, core biopsy devices have been developed to obtain histologic tissue samples using a standard linear array echoendoscope. Two such devices introduced over the last decade include the Quick-Core and ProCore biopsy needles (Cook Medical, Bloomington, IN). In a multicenter cohort study of 109 patients with intestinal and extraintestinal lesions (including 47 pancreatic tumors), the ProCore needle provided adequate histology and a correct diagnosis in $96 \%$ and $89 \%$ of cases, respectively [75]. However, in a recent metaanalysis including 9 studies of 576 patients, there was no difference in diagnostic adequacy (75\% vs $89 \%$ ), diagnostic accuracy (86\% vs $86 \%$ ), or rate of histologic core specimen acquisition (78\% vs 77\%) between the ProCore and standard FNA needles, respectively. The mean number of passes required for diagnosis, however, was significantly lower when using the ProCore needle (standardized mean difference $-1.2, P<0.001$ ) [76]. Table 2 summarizes the various published studies comparing the ProCore needle to standard FNA needles. Nevertheless, core biopsy needles will continue serving niche applications such as aiding the diagnosis of autoimmune pancreatitis (Figure 3) [77] and pancreatic lymphoma [78], where its superiority has been demonstrated in previous studies. In addition, core biopsy needles could be used as a rescue technique when on-site FNA results are inconclusive or if this service is not available (Video 2; Figure 4) [75].

\subsubsection{Ancillary studies}

In an attempt to increase the sensitivity of EUS-FNA to detect malignancy in pancreatic masses, investigators have evaluated the presence of certain genetic mutations. A meta-analysis of
Summary of studies that assessed the Procore needle for EUS-FNB compared to FNA when comparative data is available.

\begin{tabular}{|c|c|c|c|c|c|c|c|}
\hline Author & Study design & No. of patients & Needle gauge FNB & SA FNB (\%) & DY FNB (\%) & DY FNA (\%) & $P$ value \\
\hline Larghi et al [64] & Prospective cohort & 61 & 22 & 89 & 89 & $\mathrm{n} / \mathrm{a}$ & $\mathrm{n} / \mathrm{a}$ \\
\hline Iwashita et al [65] & Retrospective & 38 & 25 & $\mathrm{n} / \mathrm{a}$ & $86 / 96$ & $\mathrm{n} / \mathrm{a}$ & $\mathrm{n} / \mathrm{a}$ \\
\hline Strand et al [67] & Prospective cohort & 32 & 22 & $\mathrm{n} / \mathrm{a}$ & 28 & 93 & $<0.001$ \\
\hline Choi et al [68] & Retrospective & 80 & 22 & $\mathrm{n} / \mathrm{a}$ & 90 & 62 & $<0.005$ \\
\hline Singh et al [69] & Retrospective & 40 & 22 & $\mathrm{n} / \mathrm{a}$ & 100 & 93 & NS \\
\hline
\end{tabular}

Abbreviations: DY, diagnostic yield; FNB, Fine needle biopsy; RCT, randomized controlled trial; SA, specimen adequacy.

(Adapted with permission from Wani et al [39]). 


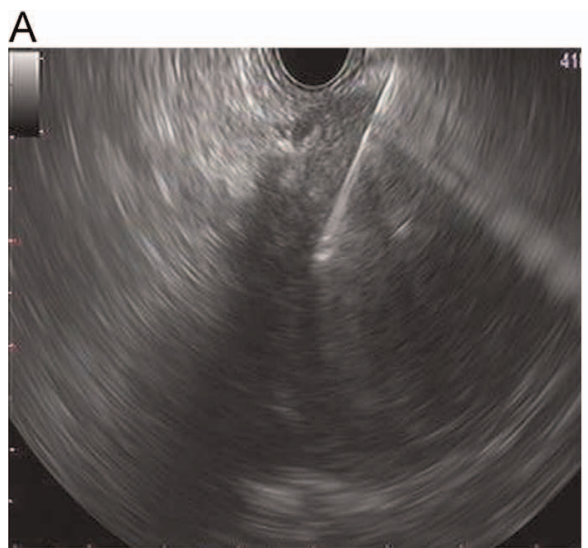

B

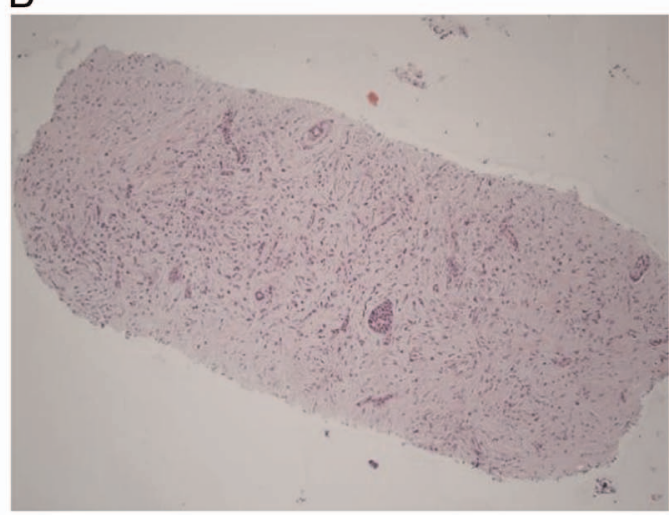

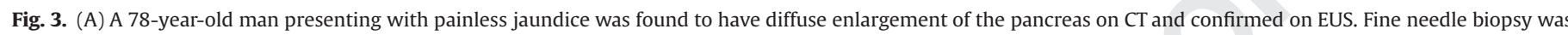

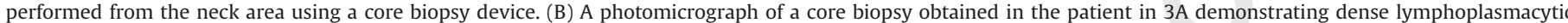

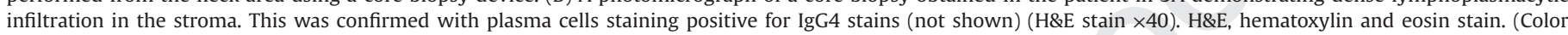
version of figure is available online.)

8 prospective studies involving 931 patients who had KRAS mutation analysis on EUS-FNA specimens reported a pooled sensitivity and specificity of $77 \%$ and $93 \%$, respectively [79]. When combined with EUS-FNA alone, the addition of k-ras mutation testing increased sensitivity from $81 \%-89 \%$ but reduced specificity from $97 \%-92 \%$.

Fluorescence in situ hybridization (FISH) processing of EUSguided FNA specimens are other assays that were found to increase the sensitivity and accuracy of routine cytology examination [80]. Furthermore, Kubiliun et al [81] showed that in patients with suspected pancreatic cancer, FISH analysis can detect additional cases missed by cytology without compromising specificity. Authors recommended EUS-FNA with rescue FISH for the diagnosis of pancreatic carcinoma in patients with inconclusive on-site cytopathology results. Finally, combining routine cytology with FISH and KRAS analyses improves diagnostic yield of EUS-FNA of solid pancreatic masses, according to Reicher et al [82]. Such assays can be included to further investigate atypical cytology from pancreatic EUS-FNA.

It should be noted though that KRAS mutations can be present in the setting of chronic pancreatitis and could lead to false positive results in $>10 \%$ of cases, however, the specificity of FISH in this setting remains high exceeding 95\% [82]. To overcome such limitations, differential miRNA expression in tissue specimens has been explored as an adjunct to cytopathology for the diagnosis and prognostication of individuals with pancreatic cancer [83,84]. A study measuring miR-10b expression in EUS-FNA tissue samples revealed an association between decreased miR-10b expression in pancreas cancer cells with improved survival, response to

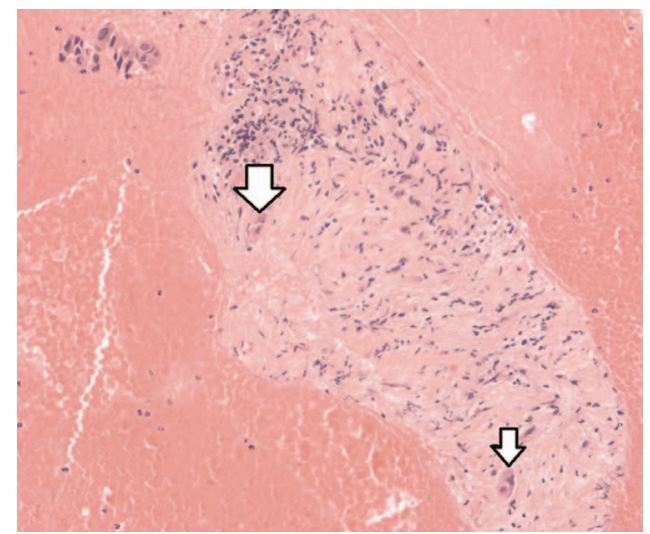

Fig. 4. $(H \& E \times 200)$ A patient with pancreatic head mass and previously inconclusive EUS-FNA. Fine needle biopsy confirmed pancreatic adenocarcinoma. Rare malignant cells can be seen in a fibrous stroma (arrows). (Color version of figure is available online.) neoadjuvant radiochemotherapy, and delayed time to metastasis [83]. Brand et al [85] developed and validated a 5-miRNA panel derived from EUS samples that were prospectively collected at multiple centers. This 5-miRNA panel can accurately predict which preoperative pancreatic EUS-FNA specimens contain PDAC. This test might aid in the diagnosis of pancreatic cancer by reducing the number of FNAs without a definitive adenocarcinoma diagnosis, thereby reducing the number of repeat EUS-FNA procedures, which could reduce procedure complications and the need for multiple needles, and provide faster times to complete EUS-FNA. As the list of known microRNAs involved in pancreatic cancer pathogenesis continues to expand, we expect the utilization of such assay to grow over the next decade and become commercially available.

\subsection{Safety of EUS and EUS-FNA}

EUS is a safe procedure with a reported overall adverse event rate of $1.1 \%-3 \%$ [86].

Two major possible adverse events of EUS-FNA of solid pancreatic masses include acute pancreatitis and the risk of needle tract seeding. The reported risk of acute pancreatitis after EUS-FNA of solid pancreatic masses is $0.26 \%-0.85 \%$ [87-89]. This risk can be decreased by minimizing the number of needle passes, minimizing the amount of normal appearing pancreatic parenchyma traversed with each pass, and avoiding needle insertion through the pancreatic duct unless it is absolutely necessary. Needle tract seeding is a consideration with biopsy of pancreatic masses, but most of the published data are limited to case reports [90]. The reported incidence of needle tract seeding after EUS-FNA is believed to be lower than percutaneous CT or transabdominal ultrasound-guided sampling (2.2\% vs $16.3 \%$ ) [91]. The majority of the reported cases of EUS-FNA needle tract seeding are for body and tail cancers, which were sampled through the gastric wall [90]. Needle tract seeding is of less significance in resectable pancreatic head tumors sampled transduodenally, because the site of needle puncture is included within the resection margins of a pancreatoduodenectomy.

\section{Pancreatic neuroendocrine tumors}

The use of EUS-FNA permits tissue confirmation of a suspected pancreatic neuroendocrine tumors (PNET) [92,93]. Data from a large retrospective case series of 80 patients, suggested that EUS should be included in the diagnostic workup of all patients with suspected PNETs, even when the CT study was negative for a primary lesion in the pancreas. 


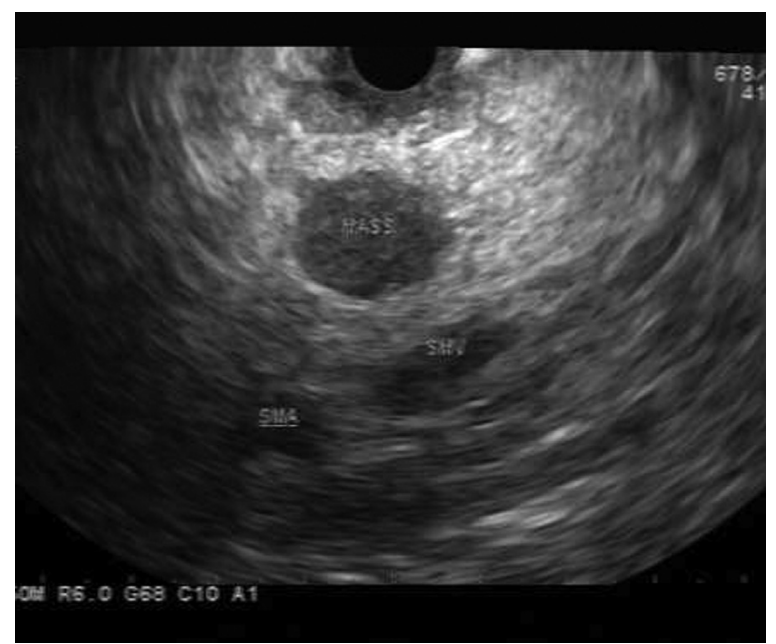

Fig. 5. A small mass in the pancreatic head in a patient with MEN1 noted on EUS screening examination. This lesion was not seen on CT scan but was confirmed to be PNET on FNA. MEN1, multiple neuroendocrine neoplasia type 1.

EUS and EUS-FNA are highly sensitive and accurate for the diagnosis of PNETs [94-96]. Characteristic EUS findings are helpful 5 for the diagnosis and grading of PNETs (Figure 5) [94,95]. However, location of the tumor in the pancreatic head and presence of rich stromal fibrosis can negatively impact sampling adequacy [96]. Purely cystic and mixed solid-cystic PNETs have distinct clinical and EUS characteristics, and are associated with less aggressive biological behavior compared with solid PNETs. EUS-FNA is accurate for determining malignant potential on preoperative evaluation. Despite complete resection, recurrence is observed up to 5 years following surgery [97]. Cytology is usually diagnostic in 6 PNETs (Figure. 6), which typically stains positively for chromogra7 nin and synaptophysin (Figure 7). Recently, molecular assays allowed genetic mutations to be reliably assessed on FNA specimens from PNETs. A recent study of 29 patients with PNETs followed for an average of 33 months showed that the presence of allelic microsatellite loss was associated with increased PNET recurrence, progression, and mortality [98].

\section{Primary pancreatic lymphoma}

EUS-FNA with flow cytometry is very accurate for PPL. In a case series of 16 patients with PPL, Khashab et al [99] reported a

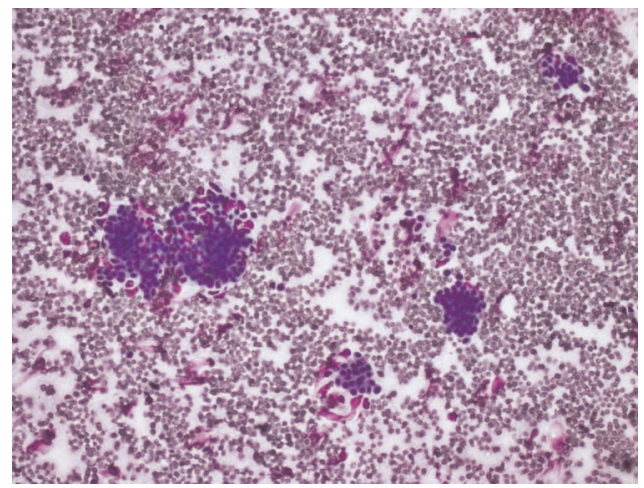

Fig. 6. FNA from a PNET demonstrating classic cytopathologic findings including predominantly small loosely cohesive groups of cells that are small to medium in size with a uniform round to oval, and often peripherally located nuclei. (H\&E stain $\times 100$ ). H\&E, hematoxylin and eosin stain. (Color version of figure is available online.)

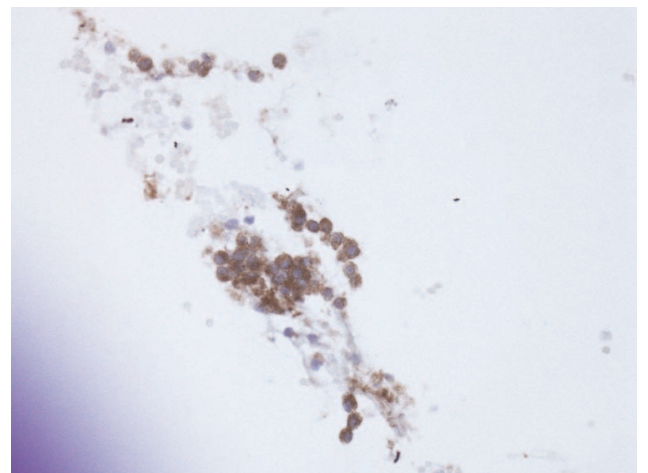

Fig. 7. Immunostains from a PNET aspirate staining positive for chromogranin (golden brown color) $(\mathrm{H} \& \mathrm{E} \times 200)$. H\&E, hematoxylin and eosin stain. (Color version of figure is available online.)

sensitivity and specificity of EUS-FNA with cytology and flow cytometry of $84.6 \%$ and $100 \%$, respectively. This is in contrast to EUS-FNA with cytology alone, which had sensitivity and specificity less than $30 \%$. This diagnosis should be suspected based on clinical appearance, lack of definite malignancy, and abundance of abnormal lymphocytes on rapid cytological review.

\section{Pancreatic metastases}

EUS-FNA permits an accurate cytologic diagnosis of metastatic lesions to the pancreas. In the largest series to date of 72 masses in 49 patients, El Hajj et al [100] reported metastatic lesions from kidney (renal cell carcinoma in 21$)$, lung $(n=8)$, skin $(n=6)$, colon $(n=4)$, breast $(n=3)$, small bowel $(n=2)$, stomach $(n=2)$, liver $(n=1)$, ovary $(n=1)$, and bladder $(n=1)$. Metastasis to the pancreas may occur many years (especially for renal cell carcinoma; Figure 8) after diagnosis of the primary tumor. Obtaining a detailed medical history for previous malignancy may raise suspicion for this diagnosis. In patients with a remote history of malignancy, obtaining additional cytological material for cell block and the use of immunocytochemistry may be helpful to confirm the diagnosis of pancreatic metastases and confirm recurrent malignancy.

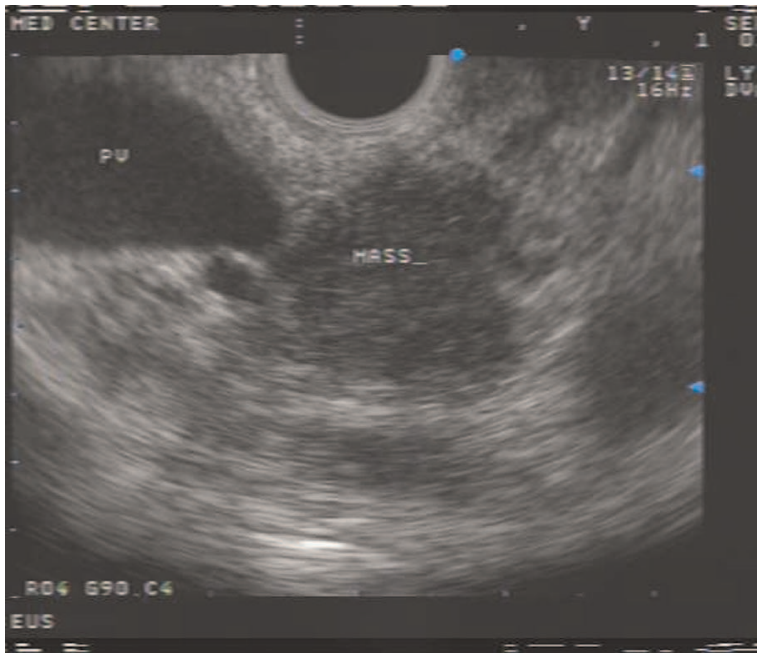

Fig. 8. A patient with known history of renal cell carcinoma treated 15 years earlier presented with painless jaundice. A pancreatic head lesion was found on EUS, confirmed on FNA to be renal cell carcinoma.
712

713 


\section{Appendix A. Supplementary material}

Supplementary data associated with this article can be found in the online version at http://dx.doi.org/10.1016/j.tgie.2018.01.002.

\section{References}

[1] Fogel EL, Shahda S, Sandrasegaran K, et al. A multidisciplinary approach to pancreas cancer in 2016: a review. Am J Gastroenterol 2017;112:537-54.

[2] ASGE Standards of Practice Committee, Eloubeidi MA, Decker GA, Chandrasekhara V, et al. The role of endoscopy in the evaluation and management of patients with solid pancreatic neoplasia. Gastrointest Endosc 2016;8317-28

[3] Al-Haddad M, DeWitt J. EUS in pancreas tumors. In: Hawes RH, Fockens P, Varadarajulu S, editors. Endosonography. 2nd ed. Philadelphia, PA: Elsevier Inc; 2011. p. 148-65.

[4] Siegel RL, Miller KD, Jemal A. Cancer statistics, 2015. CA Cancer J Clin 2015;65:5-29.

[5] Rahib L, Smith BD, Aizenberg R, et al. Projecting cancer incidence and deaths to 2030: the unexpected burden of thyroid, liver, and pancreas cancers in the United States. Cancer Res 2014;74:2913-21.

[6] Bronstein YL, Loyer EM, Kaur H, et al. Detection of small pancreatic tumors with multiphasic helical CT. AJR Am J Roentgenol 2004;182:619-23.

[7] Agarwal B, Abu-Hamda E, Molke KL, Correa AM, Ho L. Endoscopic ultrasound-guided fine needle aspiration and multidetector spiral CT in the diagnosis of pancreatic cancer. Am J Gastroenterol 2004;99:844-50.

[8] DeWitt J, Devereaux B, Chriswell M, et al. Comparison of endoscopic ultrasonography and multidetector computed tomography for detecting and staging pancreatic cancer. Ann Intern Med 2004;141:753-63.

[9] Vellet AD, Romano W, Bach DB, et al. Adenocarcinoma of the pancreatic ducts: comparative evaluation with CT and MR imaging at 1.5 T. Radiology 1992:183:87-95.

[10] Holalkere NS, Sahani DV, Blake MA, et al. Characterization of small liver lesions: added role of MR after MDCT. J Comput Assist Tomogr 2006;30: 591-6.

[11] Park HS, Lee JM, Choi HK, et al. Preoperative evaluation of pancreatic cancer: comparison of gadolinium-enhanced dynamic MRI with MR cholangiopancreatography versus MDCT. J Magn Reson Imaging 2009;30:586-95.

[12] Koelblinger C, Ba-Ssalamah A, Goetzinger P, et al. Gadobenate dimeglumineenhanced 3.0-T MR imaging versus multiphasic 64-detector row CT: prospective evaluation in patients suspected of having pancreatic cancer. Radiology 2011:259:757-66.

[13] Tempero MA, Malafa MP, Behrman SW, et al. Pancreatic adenocarcinoma, version 2.2014: featured updates to the NCCN guidelines. J Natl Compr Canc Netw 2014;12:1083-93.

[14] Strobel K, Heinrich S, Bhure U, et al. Contrast-enhanced 18F-FDG PET/CT: 1 -stop-shop imaging for assessing the resectability of pancreatic cancer. J Nucl Med 2008;49:1408-13.

[15] Sendler A, Avril N, Helmberger H, et al. Preoperative evaluation of pancreatic masses with positron emission tomography using 18F-fluorodeoxyglucose: diagnostic limitations. World J Surg 2000;24:1121-9.

[16] Kitajima K, Murakami K, Yamasaki E, et al. Performance of integrated FDGPET/contrast-enhanced CT in the diagnosis of recurrent pancreatic cancer: comparison with integrated FDG-PET/non-contrast-enhanced CT and enhanced CT. Mol Imaging Biol 2010;12:452-9.

[17] Tang S, Huang G, Liu J, Liu T, et al. Usefulness of 18F-FDG PET, combined FDGPET/CT and EUS in diagnosing primary pancreatic carcinoma: a metaanalysis. Eur J Radiol 2011;78:142-50.

[18] Bilimoria KY, Bentrem DJ, Ko CY, et al. Validation of the 6th edition AJCC pancreatic cancer staging system: report from the National Cancer Database. Cancer 2007;110:738-44.

[19] Soriano A, Castells A, Ayuso C, et al. Preoperative staging and tumor resectability assessment of pancreatic cancer: prospective study comparing endoscopic ultrasonography, helical computed tomography, magnetic resonance imaging, and angiography. Am J Gastroenterol 2004;99:492-501.

[20] Tellez-Avila FI, Chavez-Tapia NC, Lopez-Arce G, et al. Vascular invasion in pancreatic cancer: predictive values for endoscopic ultrasound and computed tomography imaging. Pancreas 2012;41:636-8.

[21] Shami VM, Mahajan A, Loch MM, et al. Comparison between endoscopic ultrasound and magnetic resonance imaging for the staging of pancreatic cancer. Pancreas 2011;40:567-70.

[22] Hajj El II, Eloubeidi M. Sampling para-aortic lymph nodes in pancreatic and biliary cancers with EUS-guided FNA: diagnostic, clinical, and therapeutic implications. Gastrointest Endosc 2016;84:476-8 (editorial).

[23] Kurita A, Kodama Y, Nakamoto Y, et al. Impact of EUS-FNA for preoperative para-aortic lymph node staging in patients with pancreatobiliary cancer. Gastrointest Endosc 2016;84:467-75.

[24] DeWitt J, Yu M, Al-Haddad MA, et al. Survival in patients with pancreatic cancer after the diagnosis of malignant ascites or liver metastases by EUSFNA. Gastrointest Endosc 2010;71:260-5.

[25] Gress FG, Hawes RH, Savides TJ, et al. Role of EUS in the preoperative staging of pancreatic cancer: a large single-center experience. Gastrointest Endosc 1999;50:786-91.
[26] Ramsay D, Marshall M, Song S, et al. Identification and staging of pancreatic tumours using computed tomography, endoscopic ultrasound and mangafodipir trisodium-enhanced magnetic resonance imaging. Australas Radiol 2004;48:154-61.

[27] Buscail L, Pages P, Berthelemy P, et al. Role of EUS in the management of pancreatic and ampullary carcinoma: a prospective study assessing resectability and prognosis. Gastrointest Endosc 1999;50:34-40.

[28] Rosch T, Braig C, Gain T, et al. Staging of pancreatic and ampullary carcinoma by endoscopic ultrasonography. Comparison with conventional sonography, computed tomography, and angiography. Gastroenterology 1992;102: 188-99.

[29] Rosch T, Dittler HJ, Strobel K, et al. Endoscopic ultrasound criteria for vascular invasion in the staging of cancer of the head of the pancreas: a blind reevaluation of videotapes. Gastrointest Endosc 2000;52:469-77.

[30] Sugiyama M, Hagi H, Atomi Y, Saito M. Diagnosis of portal venous invasion by pancreatobiliary carcinoma: value of endoscopic ultrasonography. Abdom Imaging 1997;22:434-8.

[31] Midwinter MJ, Beveridge CJ, Wilsdon JB, et al. Correlation between spiral computed tomography, endoscopic ultrasonography and findings at operation in pancreatic and ampullary tumours. Br J Surg 1999;86:189-93.

[32] Mertz HR, Sechopoulos P, Delbeke D, et al. EUS, PET, and CT scanning fo evaluation of pancreatic adenocarcinoma. Gastrointest Endosc 2000;52: $367-71$.

[33] Ahmad NA, Lewis JD, Siegelman ES, et al. Role of endoscopic ultrasound and magnetic resonance imaging in the preoperative staging of pancreatic adenocarcinoma. Am J Gastroenterol 2000;95:1926-31.

[34] Howard TJ, Chin AC, Streib EW, et al. Value of helical computed tomography, angiography, and endoscopic ultrasound in determining resectability of periampullary carcinoma. Am J Surg 1997;174:237-41.

[35] Legmann P, Vignaux O, Dousset B, et al. Pancreatic tumors: comparison of dual-phase helical CT and endoscopic sonography. AJR Am J Roentgenol 1998:170:1315-22.

[36] Tierney WM, Francis IR, Eckhauser F, et al. The accuracy of EUS and helical CT in the assessment of vascular invasion by peripapillary malignancy. Gastrointest Endosc 2001;53:182-8.

[37] Bao PQ Johnson JC, Lindsey EH, et al. Endoscopic ultrasound and computed tomography predictors of pancreatic cancer resectability. J Gastrointest Surg 2008; 12:10-6.

[38] Singh A, Faulx AL. Endoscopic evaluation in the workup of pancreatic cancer Surg Clin North Am 2016;96:1257-70.

[39] Wani S, Muthusamy VR, Komanduri S. EUS-guided tissue acquisition: an evidence-based approach (with videos). Gastrointest Endosc 2014;80: 939-59.

[40] Hewitt MJ, McPhail MJ, Possamai L, et al. EUS-guided FNA for diagnosis of solid pancreatic neoplasms: a meta-analysis. Gastrointest Endosc 2012;75: 319-31.

[41] Chen G, Liu S, Zhao Y, et al. Diagnostic accuracy of endoscopic ultrasoundguided fine-needle aspiration for pancreatic cancer: a meta-analysis. Pancreatology 2013:13:298-304.

[42] Fritscher-Ravens A, Brand L, Knofel WT, et al. Comparison of endoscopic ultrasound-guided fine needle aspiration for focal pancreatic lesions in patients with normal parenchyma and chronic pancreatitis. Am J Gastroenterol 2002:97:2768-75.

[43] Wani S, Shah RJ. EUS-guided tissue acquisition: do we need to shoot for a "core" to score? Gastrointest Endosc 2016;84:1047-9.

[44] Attam R, Arain MA, Bloechl SJ, et al. "Wet suction technique (WEST)": a novel way to enhance the quality of EUS-FNA aspirate. Results of a prospective, single-blind, randomized, controlled trial using a 22-gauge needle for EUSFNA of solid lesions. Gastrointest Endosc 2015;81:1401-7.

[45] Berzosa M, Villa N, Bartel MJ, et al. Pilot study comparing hybrid vs. wet vs, dry suction techniques for EUS-FNA of solid lesions. Gastrointest Endosc 2014;79:AB430.

[46] Kudo T, Kawakami H, Hayashi T, et al. High and low negative pressure suction techniques in EUS-guided fine-needle tissue acquisition by using $25-$ gauge needles: a multicenter, prospective, randomized, controlled trial. Gastrointest Endosc 2014:80:1030-7.

[47] Chen AM, Park WG, Friedland S, et al. Endoscopic ultrasound-guided fine needle aspiration versus fine needle capillary sampling biopsy of pancreatic solid lesions: does technique matter? Gastrointest Endosc 2011:73:AB331.

[48] Nakai Y, Isayama $\mathrm{H}$, Chang KJ, et al. Slow pull versus suction in endoscopic ultrasound-guided fine-needle aspiration of pancreatic solid masses. Dig Dis Sci 2014;59:1578-85.

[49] Chen AM, Thosani NC, Friedland S, et al. Prospective randomized blind controlled trial of capillary EUS-FNA vs. suction EUS-FNA for the diagnosis of solid tumors. Gastrointest Endosc 2014;79:AB111.

[50] Wani S, Early D, Kunkel J, et al. Diagnostic yield of malignancy during EUSguided FNA of solid lesions with and without a stylet: a prospective, single blind, randomized, controlled trial. Gastrointest Endosc 2012;76:328-35.

[51] Affolter KE, Schmidt RL, Matynia AP, et al. Needle size has only a limited effect on outcomes in EUS-guided fine needle aspiration: a systematic review and meta-analysis. Dig Dis Sci 2013;58:1026-34.

[52] Madhoun MF, Wani SB, Rastogi A, et al. The diagnostic accuracy of 22-gauge and 25-gauge needles in endoscopic ultrasound-guided fine needle aspiration of solid pancreatic lesions: a meta-analysis. Endoscopy 2013;45:86-92.

[53] Xu MM, Jia HY, Yan LL, et al. Comparison of two different size needles in endoscopic ultrasound-guided fine-needle aspiration for diagnosing solid 
pancreatic lesions: a meta-analysis of prospective controlled trials. Medicine (Baltimore) 2017:96:e5802.

[54] Itoi T, Itokawa F, Sofuni A, et al. Puncture of solid pancreatic tumors guided by endoscopic ultrasonography: a pilot study series comparing trucut and 19-gauge and 22-gauge aspiration needles. Endoscopy 2005;37:362-6.

[55] Song TJ, Kim JH, Lee SS, et al. The prospective randomized, controlled trial of endoscopic ultrasound-guided fine-needle aspiration using $22 \mathrm{G}$ and $19 \mathrm{G}$ aspiration needles for solid pancreatic or peripancreatic masses. Am J Gastroenterol 2010;105:1739-45.

[56] Siddiqui UD, Rossi F, Rosenthal LS, et al. EUS-guided FNA of solid pancreatic masses: a prospective, randomized trial comparing 22-gauge and 25-gauge needles. Gastrointest Endosc 2009;70:1093-7.

[57] Yusuf TE, Ho S, Pavey DA, et al. Retrospective analysis of the utility of endoscopic ultrasound-guided fine-needle aspiration (EUS-FNA) in pancreatic masses, using a 22-gauge or 25-gauge needle system: a multicenter experience. Endoscopy 2009;41:445-8.

[58] Siddiqui AA, Lyles T, Avula H, et al. Endoscopic ultrasound-guided fine needle aspiration of pancreatic masses in a veteran population: comparison of results with 22- and 25-gauge needles. Pancreas 2010;39:685-6.

[59] Camellini L, Carlinfante G, Azzolini F, et al. A randomized clinical trial comparing $22 \mathrm{G}$ and $25 \mathrm{G}$ needles in endoscopic ultrasound-guided fineneedle aspiration of solid lesions. Endoscopy 2011;43:709-15.

[60] Uehara H, Ikezawa K, Kawada N, et al. Diagnostic accuracy of endoscopic ultrasound-guided fine needle aspiration for suspected pancreatic malignancy in relation to the size of lesions. J Gastroenterol Hepatol 2011;26:1256-61.

[61] Fabbri C, Polifemo AM, Luigiano C, et al. Endoscopic ultrasound-guided fine needle aspiration with 22- and 25-gauge needles in solid pancreatic masses: a prospective comparative study with randomisation of needle sequence. Dig Liver Dis 2011:43:647-52.

[62] Bang JY, Magee SH, Ramesh J, et al. Randomized trial comparing fanning with standard technique for endoscopic ultrasound-guided fine-needle aspiration of solid pancreatic mass lesions. Endoscopy 2013:45:445-50.

[63] Iwashita T, Nakai Y, Samarasena JB, et al. High single-pass diagnostic yield of a new 25-gauge core biopsy needle for EUS-guided FNA biopsy in solid pancreatic lesions. Gastrointest Endosc 2013;77:909-15.

[64] Larghi A, Iglesias-Garcia J, Poley JW, et al. Feasibility and yield of a novel 22gauge histology EUS needle in patients with pancreatic masses: a multicenter prospective cohort study. Surg Endosc 2013;27:3733-8.

[65] Bang JY, Hebert-Magee S, Trevino J, et al. Randomized trial comparing the 22-gauge aspiration and 22-gauge biopsy needles for EUS-guided sampling of solid pancreatic mass lesions. Gastrointest Endosc 2012;76:321-7.

[66] Vanbiervliet G, Fumex F, Saint-Paul MC, et al. Prospective randomized controlled trial with crossover of endoscopic ultrasound fine needle aspiration (EUS-FNA) using 22G ProCore and 22G EchoTip Needle for solid pancreatic mass: the "Picore" study [abstract]. Gastrointest Endosc 2013;77: AB178.

[67] Strand DS, Shami VM, Sauer BG, et al. EUS-guided 22-gauge fine needle aspiration is superior to EUS-guided 22-gauge core needle biopsy in the evaluation of solid pancreatic neoplasms [abstract]. Gastrointest Endosc 2013;77:AB1403.

[68] Choi HJ, Jong HM, Hee KK, et al. Comparison of EUS-fine needle biopsy with EUS-fine needle aspiration as a historical control for diagnosis of pancreatic solid masses [abstract]. Gastrointest Endosc. 2013;77:AB401.

[69] Singh M, Ramay F, Sood V. EUS FNA/FNB of pancreatic mass lesions-single tertiary referral center experience [abstract]. Gastrointest Endosc 2013;77: AB402.

[70] Hébert-Magee S, Bae S, Varadarajulu S, et al. The presence of a cytopathologist increases the diagnostic accuracy of endoscopic ultrasound-guided fine needle aspiration cytology for pancreatic adenocarcinoma: a meta-analysis. Cytopathology 2013;24:159-71.

[71] Wani S, Mullady D, Early DS, et al. The clinical impact of immediate on-site cytopathology evaluation during endoscopic ultrasound-guided fine needle aspiration of pancreatic masses: a prospective multicenter randomized controlled trial. Am J Gastroenterol 2015;110:1429-39.

[72] Lee LS, Nieto J, Watson RR, et al. Randomized noninferiority trial comparing diagnostic yield of cytopathologist-guided versus 7 passes for EUS-FNA of pancreatic masses. Dig Endosc 2015 [Epub ahead of print].

[73] Mounzer R, Yen R, Marshall C, et al. Interobserver agreement among cytopathologists in the evaluation of pancreatic endoscopic ultrasoundguided fine needle aspiration cytology specimens. Endosc Int Open 2016;4: E812-9.

[74] Wani S, Hall M, Keswani RN, et al. Variation in aptitude of trainees in endoscopic ultrasonography, based on cumulative sum analysis. Clin Gastroenterol Hepatol 2015;13:1318-25.

[75] Iglesias-Garcia J, Poley JW, Larghi A, et al. Feasibility and yield of a new EUS histology needle: results from a multicenter, pooled, cohort study. Gastrointest Endosc 2011;73:1189-96.

[76] Bang JY, Hawes R, Varadarajulu S. A meta-analysis comparing ProCore and standard fine-needle aspiration needles for endoscopic ultrasound-guided tissue acquisition. Endoscopy 2016;48:339-49.
[77] Levy MJ, Reddy RP, Wiersema MJ, et al. EUS-guided trucut biopsy in establishing autoimmune pancreatitis as the cause of obstructive jaundice. Gastrointest Endosc 2005;61:467-72.

[78] Eloubeidi MA, Mehra M, Bean SM. EUS-guided 19-gauge trucut needle biopsy for diagnosis of lymphoma missed by EUS-guided FNA. Gastrointest Endosc 2007;65:937-9.

[79] Fuccio L, Hassan C, Laterza L, et al. The role of K-ras gene mutation analysis in EUS-guided FNA cytology specimens for the differential diagnosis of pancreatic solid masses: a meta-analysis of prospective studies. Gastrointest Endosc 2013;78:596-608.

[80] Levy MJ, Clain JE, Clayton A, et al. Preliminary experience comparing routine cytology results with the composite results of digital image analysis and fluorescence in situ hybridization in patients undergoing EUS-guided FNA. Gastrointest Endosc 2007:66:483-90.

[81] Kubiliun N, Ribeiro A, Fan YS, et al. EUS-FNA with rescue fluorescence in situ hybridization for the diagnosis of pancreatic carcinoma in patients with inconclusive on-site cytopathology results. Gastrointest Endosc 2011;74: $541-7$.

[82] Reicher S, Boyar FZ, Albitar M, et al. Fluorescence in situ hybridization and K-ras analyses improve diagnostic yield of endoscopic ultrasound-guided fine-needle aspiration of solid pancreatic masses. Pancreas 2011;40: $1057-62$.

[83] Preis M, Gardner TB, Gordon SR, et al. MicroRNA-10b expression correlates with response to neoadjuvant therapy and survival in pancreatic ductal adenocarcinoma. Clin Cancer Res 2011;17:5812-21.

[84] Sina M, Coté GA, Korc M. Improving the diagnostic accuracy of endoscopic ultrasound-guided fine-needle aspiration using microRNAs. Gastroenterology 2014;147:930-2.

[85] Brand RE, Adai AT, Centeno BA, et al. A microRNA-based test improves endoscopic ultrasound-guided cytologic diagnosis of pancreatic cancer. Clin Gastroenterol Hepatol 2014;12:1717-23.

[86] Adler DG, Jacobson BC, Davila RE, et al. ASGE guideline: complications of EUS. Gastrointest Endosc 2005;61:8-12.

[87] Eloubeidi MA, Gress FG, Savides TJ, et al. Acute pancreatitis after EUS-guided FNA of solid pancreatic masses: a pooled analysis from EUS centers in the United States. Gastrointest Endosc 2004;60:385-9.

[88] Eloubeidi MA, Tamhane A, Varadarajulu S, et al. Frequency of major complications after EUS-guided FNA of solid pancreatic masses: a prospective evaluation. Gastrointest Endosc 2006;63:622-9.

[89] Al-Haddad M, Wallace MB, Woodward TA, et al. The safety of fine-needle aspiration guided by endoscopic ultrasound: a prospective study. Endoscopy 2008;40:204-8.

[90] Tomonari A, Katanuma A, Matsumori T, et al. Resected tumor seeding in stomach wall due to endoscopic ultrasonography-guided fine needle aspiration of pancreatic adenocarcinoma. World J Gastroenterol 2015;21:8458-61.

[91] Micames C, Jowell PS, White R, et al. Lower frequency of peritoneal carcinomatosis in patients with pancreatic cancer diagnosed by EUS-guided FNA vs. percutaneous FNA. Gastrointest Endosc 2003;58:690-5.

[92] Manta R, Nardi E, Pagano N, et al. Pre-operative diagnosis of pancreatic neuroendocrine tumors with endoscopic ultrasonography and computed tomography in a large series. J Gastrointest Liver Dis 2016;25:317-21.

[93] Mitra V, Nayar MK, Leeds JS, et al. Diagnostic performance of endoscopic ultrasound (EUS)/endoscopic ultrasound-fine needle aspiration (EUS-FNA) cytology in solid and cystic pancreatic neuroendocrine tumours. J Gastrointest Liver Dis 2015;24:69-75.

[94] Fujimori N, Osoegawa T, Lee L, et al. Efficacy of endoscopic ultrasonography and endoscopic ultrasonography-guided fine-needle aspiration for the diagnosis and grading of pancreatic neuroendocrine tumors. Scand J Gastroenterol 2016;51:245-52.

[95] Unno J, Kanno A, Masamune A, et al. The usefulness of endoscopic ultrasound-guided fine-needle aspiration for the diagnosis of pancreatic neuroendocrine tumors based on the World Health Organization classification. Scand J Gastroenterol 2014;49:1367-74.

[96] Hijioka S, Hara K, Mizuno N, et al. Diagnostic performance and factors influencing the accuracy of EUS-FNA of pancreatic neuroendocrine neoplasms. J Gastroenterol 2016;51:923-30.

[97] Ridtitid W, Halawi H, DeWitt JM, et al. Cystic pancreatic neuroendocrine tumors: outcomes of preoperative endosonography-guided fine needle aspiration, and recurrence during long-term follow-up. Endoscopy 2015;47: $617-25$.

[98] Fasanella KE, McGrath KM, Sanders M, et al. Pancreatic endocrine tumor EUS-guided FNA DNA microsatellite loss and mortality. Gastrointest Endosc 2009;69:1074-80.

[99] Khashab M, Mokadem M, DeWitt J, et al. Endoscopic ultrasound-guided fineneedle aspiration with or without flow cytometry for the diagnosis of primary pancreatic lymphoma-a case series. Endoscopy 2010;42:228-31.

[100] El Hajj II, Leblanc JK, Sherman S, et al. Endoscopic ultrasound-guided biopsy of pancreatic metastases: a large single-center experience. Pancreas 2013;42: $524-30$. 